\title{
Johann Friedrich Beyschlags Dissertation von 1734 über das fossile Elfenbein von Schwäbisch Hall
}

\author{
von Walther Ludwig und Hellmar Weber
}

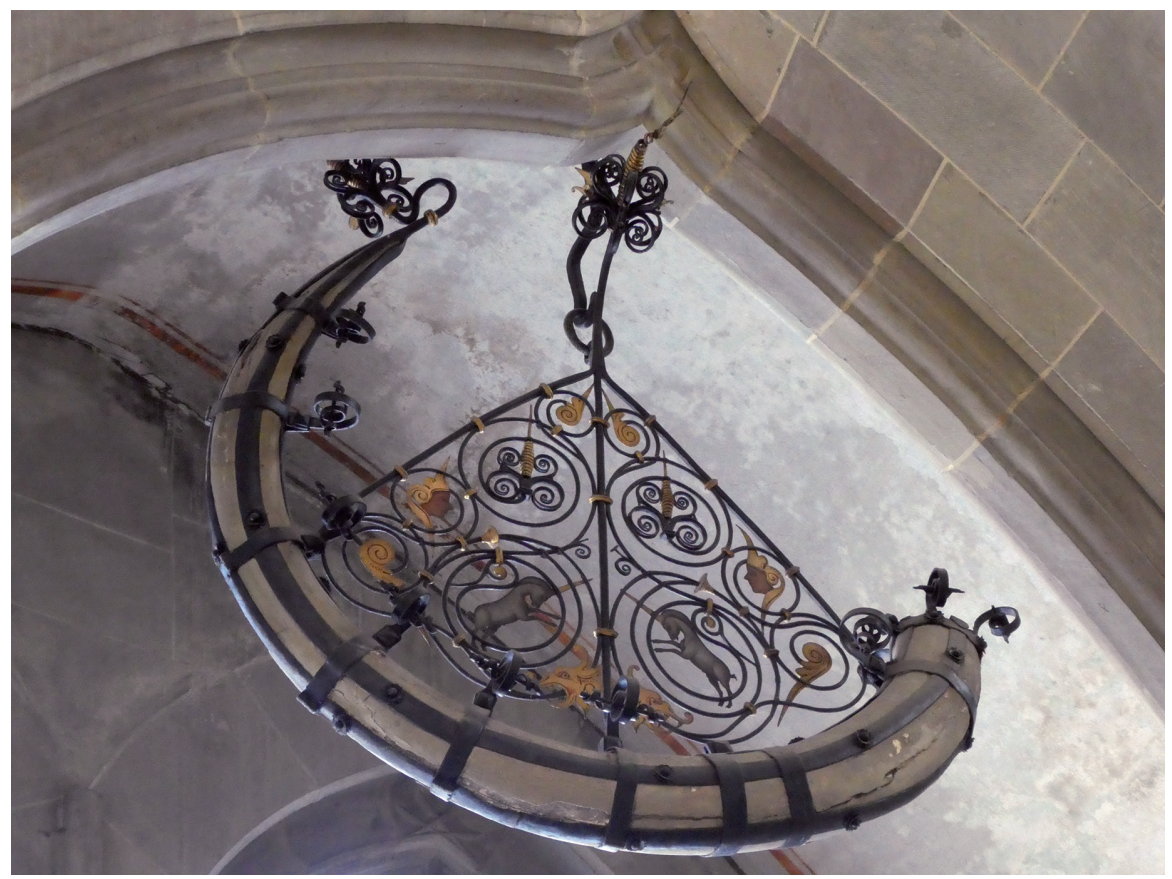

Abb. 1 Mammutstoßzahn in schmiedeeiserner Fassung im Chor der Kirche St. Michael in Schwäbisch Hall, gefunden 1605 bei Hohenstadt im Bühlertal. Foto: Hellmar Weber, 18. Januar 2017.

Ein Stich (Abb. 2, unten) zeigt den am 13. Februar 1605 bei Schwäbisch Hall an der Bühler gefundenen und seit Beginn des 17. Jahrhunderts im Chor der Kirche St. Michael in Schwäbisch Hall hängenden Mammutstoßzahn. Zwei Frauen, am linken bzw. rechten Rand sitzend, repräsentieren die Wissenschaften der Astronomie (Physik) und Medizin. Die linke hat einen Himmelsglobus mit dem Band der Tierkreiszeichen im Arm und berührt ein (Boylesches) Aräometer, die rechte hält ein Harnglas in der einen und einen von einer Schlange umwundenen Stab (Äskulapstab) in der anderen Hand. In Details weicht die Darstellung der kunst- 


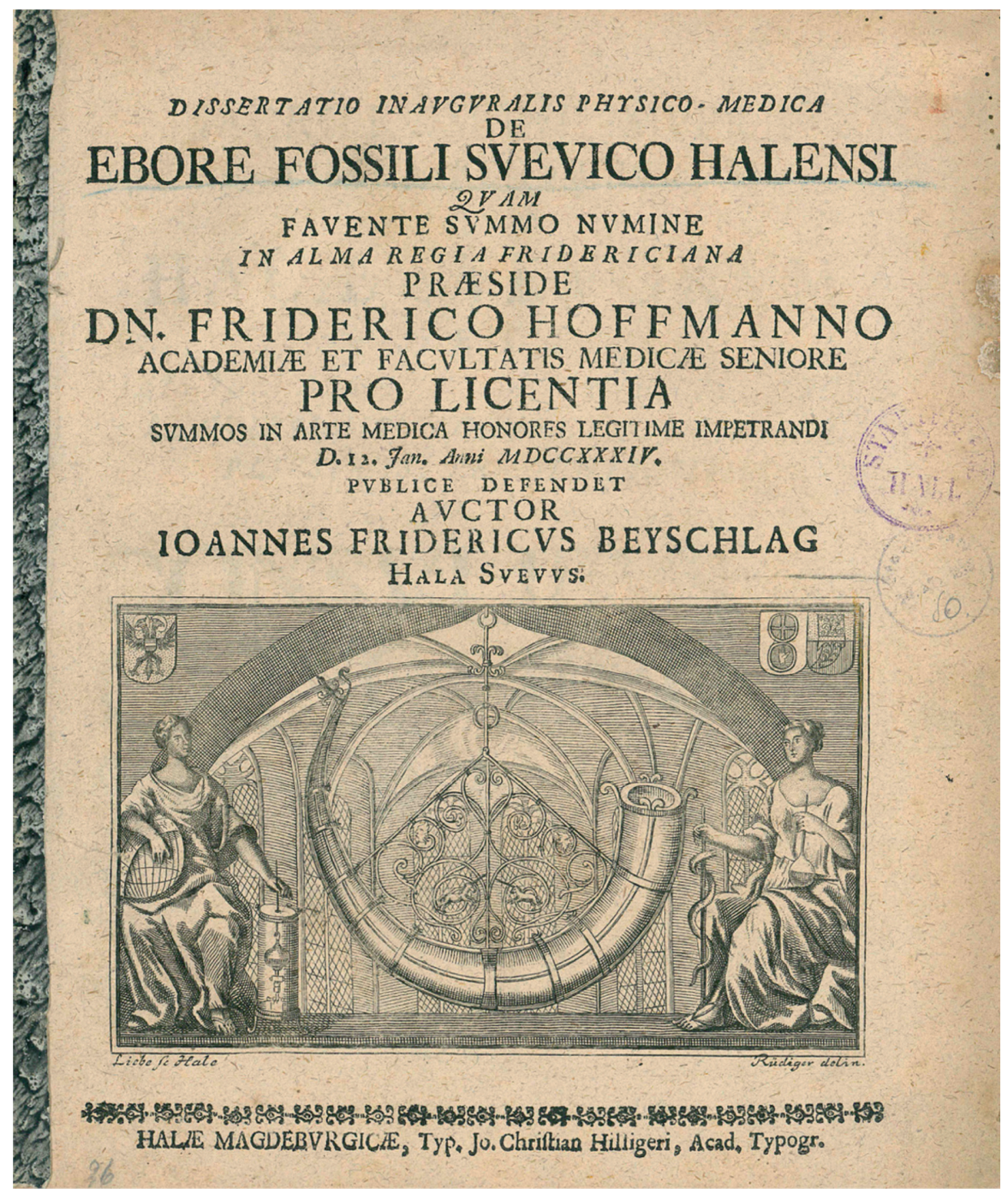

Abb. 2 Titelseite von Beyschlags Dissertation (Exemplar des Stadtarchivs Schwäbisch Hall, Signatur So 1726).

vollen schmiedeeisernen, teilweise vergoldeten Aufhängung vom Original (Abb.1) ab. Unter dem Kupferstich steht links: Liebe 1 sc[ulpsit] Halae, rechts: Rüdiger ${ }^{2}$ delin [eavit]. ${ }^{3}$

$1 \mathrm{Zu}$ Christian Gottlieb (auch Gottlieb August) Liebe, Universitätskupferstecher in Halle an der 
Titelseite der Dissertation:

$[1]^{4}$

PHYSIKALISCH-MEDIZINISCHE INAUGURALDISSERTATION

ÜBER

DAS FOSSILE SCHWÄBISCH HALLER ELFENBEIN, $D I E$

MIT GOTTES SEGEN

IN DER KÖNIGLICHEN FRIEDRICHS-UNIVERSITÄT

UNTER DEM VORSITZ

DES HERRN FRIEDRICH HOFFMANN, DEM ÄLTESTEN IN DER UNIVERSITÄT UND DER MEDIZINISCHEN FAKULTÄT, FÜR DIE ERLAUBNIS, DIE HÖCHSTEN EHREN IN DER MEDIZINISCHEN WISSENSCHAFT RECHTMÄSSIG ZU ERWERBEN, am 12. Tag des Januar des Jahres 1734. ÖFFENTLICH VERTEIDIGEN WIRD

DER VERFASSER JOHANN FRIEDRICH BEYSCHLAG AUS SCHWÄBISCH HALL.

IM MAGDEBURGISCHEN HALLE, mit den Typen des Universitätsdruckers Johann Christian Hilliger.

Im folgenden ${ }^{6}$ wird die 1734 an der Universität Halle an der Saale von dem aus Schwäbisch Hall gebürtigen Johann Friedrich Beyschlag (1710-1779) verteidig-

Saale, siehe: Allgemeines Künstlerlexikon, oder: Kurze Nachricht von dem Leben und den Werken der Maler, ...Zweyter Theil, Vierter Abschnitt. Zürich 1809, [D, s. Anm. 4], S. 703.

2 Zu Johann Anton Rüdiger, Maler in Halle an der Saale, siehe: Neues allgemeines Künstler-Lexicon oder Nachrichten von dem Leben und den Werken der Maler, ... Bearb. von G. K. Nagler. 14. Band. München 1845, [D], S. 9.

3 Liebe stach in Halle, Rüdiger zeichnete.

4 Mit [1] bis [32] wird die Seitennummerierung des ursprünglichen lateinischen Drucks hinzugefügt. In Beyschlags Druckschrift im Format 17 x $20 \mathrm{~cm}$ sind nur die Seiten 6 bis 29 mit Seitenzahlen versehen. Wir danken Herrn Dr. Andreas Maisch und Herrn Daniel Stihler (Stadt- und Hospitalarchiv Schwäbisch Hall), die uns den Druck der Beyschlagschen Arbeit zugänglich gemacht und die Abbildungen 2 und 3 angefertigt haben. Ferner geht unser Dank an Frau Wiltrud Haug-Weber für die kritische Durchsicht des Manuskripts. - Häufig von Beyschlag verwendete Abkürzungen: Lib., L., lib., l.: liber (Buch); Cap., C., cap., c.: caput (Kapitel); pag., p.: pagina (Seite); weitere Abkürzung: [D]: Digitalisat.

5 Gemeint ist die Universität in Halle an der Saale, die 1694 von dem brandenburgischen Kurfürsten Friedrich III. gegründet wurde.

6 Auf Wunsch des Erstautors wird die alte Rechtschreibung verwendet. 
te lateinische Dissertation in einer deutschen Übersetzung ediert und erläutert. ${ }^{7}$ Beyschlags Dissertation hat nicht nur regionalgeschichtliche Bedeutung, sondern gibt auch ein anschauliches Bild des damaligen naturwissenschaftlichen und medizinischen Denkens. Für die Geschichte der Paläontologie stellt sie darüber hinaus eine wertvolle Quelle dar. Die Bedeutung, die der Dissertation bei ihrem Erscheinen beigemessen wurde, belegt eine deutsche Zusammenfassung durch den Vorsitzenden der Disputation, Friedrich Hoffmann (1660-1742), die bereits wenige Jahre nach ihrer Veröffentlichung erschien. ${ }^{8} \mathrm{Da} \beta$ diese Dissertation aber von dem Kandidaten Beyschlag und nicht, wie es manchmal geschah, von dem Vorsitzenden der Disputation verfaßt wurde, beweist Hoffmanns Stellungnahme, die dem Druck der Dissertation am Ende beigegeben ist. Hier wird nun erstmals eine vollständige Übersetzung von Beyschlags Schrift vorgelegt. ${ }^{9}$ Sicher kannte und bestaunte Johann Friedrich Beyschlag seit früher Jugend den kunstvoll eingefaßten Mammutstoßzahn, der in der zentralen Chorkapelle der Schwäbisch Haller Kirche St. Michael hing und noch heute hängt (Abb. 1). ${ }^{10}$ War er doch der jüngste Sohn von Johann Balthasar Beyschlag (1669-1717), der seit 1704, zuletzt ab 1716 als Prediger und Dekan an dieser Kirche wirkte. Johann Friedrich besuchte das Haller Gymnasium illustre (Aufnahme am 20. Ok-

7 Der Anstoß zu der vorliegenden Arbeit kam von Hellmar Weber, Schwäbisch Hall. Die Übersetzung der lateinischen Dissertation wurde hergestellt von Walther Ludwig, Hamburg, der in ihrer Erläuterung von Hellmar Weber unterstützt wurde. Der Übersetzung liegt eine Kopie des Exemplars der Dissertation zugrunde, das sich im Stadtarchiv Schwäbisch Hall unter der Signatur So 1726 befindet. Die Bayerische Staatsbibliothek München verzeichnet ein anderes Exemplar, das auch online verfügbar ist: http://www.mdz-nbn-resolving.de/urn/resolver.pl?urn=urn:nbn:de:bvb:12-bsb106433 55-1; ein weiteres Digitalisat ist zu finden unter: https://books.google.com/books?id=6UIF p50I99cC \{Stand April 2017\}. Alles innerhalb der Übersetzung in eckigen Klammern Geschriebene sind Ergänzungen oder Zusätze von Walther Ludwig oder Hellmar Weber. Alle Anmerkungen wurden dem übersetzten Text hinzugefügt.

8 Friedrich Hoffmann: Von dem zu Schwäbisch Halle gegrabnen Elfenbein. In: Gründliche Auszüge, aus Medicinisch-Physicalisch- und Mathematischen Disputationibus, welche auf den Hohen Schulen sonderlich in Teutschland gehalten worden, Des anderen Bandes Erstes Stück. Leipzig 1738, [D], S. 52-60. Die S. 5 bis 19 des (lateinischen) Textes von Beyschlags Dissertation sind, ohne daß Beyschlag als Autor genannt wird, abgedruckt bei Friedrich Hoffmann: Operum omnium physico-medicorum, supplementum secundum, pars prima. Genf 1760, [D], S. 13-19. Zu Friedrich Hoffmann (1660-1742), einem im 18. Jahrhundert berühmten Arzt, vgl. August Hirsch: Hoffmann, Friedrich. In: Allgemeine Deutsche Biographie, Bd. 12. Leipzig 1880, S. 584-588, und Hans Heinz Eulner: Hoffmann, Friedrich. In: Neue Deutsche Biographie, Bd. 9. Berlin 1972, S. 416-418.

9 Bei dem im Stadtarchiv aufbewahrten Druck (wie Anm. 7) findet sich auch eine handschriftliche Übersetzung (in Sütterlinschrift) der ersten acht Seiten von Beyschlags Dissertation, deren Verfasser nicht ermittelt werden konnte. Jedenfalls kommen - das zeigt der Handschriftenvergleich - weder Wilhelm Hommel (1886-1976) noch Georg Lenckner (1888-1973), die beide im Stadtarchiv Schwäbisch Hall tätig waren, in Betracht. Möglicherweise stammt das Manuskript von einem Haller Pfarrer oder einem (Latein-)Lehrer des Haller Gymnasium illustre bzw. der späteren Lateinschule, Oberrealschule, Gymnasium, dazu: Hellmar Weber (Hrsg.): Gymnasium bei St. Michael Schwäbisch Hall. Acht Jahrhunderte Schulgeschichte in Text und Bild. Schwäbisch Hall 2014.

10 Dazu Eduard Krüger: Schriftdenkmale am Michaels-Münster zu Schwäbisch Hall. In: WFr 47 (1963), S. 59-71, hier: S. 69-71; Stefan Laube: Von der Reliquie zum Ding. Heiliger Ort - Wunderkammer - Museum. Berlin 2011, S. 110-112. 
tober 1717) ${ }^{11}$ und studierte Medizin in Jena und Halle an der Saale (immatrikuliert am 8. Mai 1730). Nach dem Studium ließ er sich als Stadtarzt (Licentiatus Physicus) in Hall nieder und heiratete 1736 die angesehene Witwe des reichen Apothekers Johann David Stellwag, dessen Familie die Haller Löwenapotheke von 1608 bis 1734 besaß. Maria Elisabeth Stellwag (1698-1770), 12 Jahre älter als Johann Friedrich - zum Zeitpunkt der Eheschließung ist sie 38 Jahre alt -, war die Tochter des Ratsherrn und fünfmaligen Stättmeisters Wolfgang Kaspar Sanwald (1669-1734). Aus erster Ehe brachte sie zwei Töchter und einen Sohn mit, aus der zweiten Ehe stammen eine Tochter und ein Sohn, der bald nach der Geburt starb. Johann Friedrich brachte es zu großem Wohlstand: 1750 war er mit 21600 Gulden drittreichster Haller Bürger. ${ }^{12}$

\section{Übersetzung der Dissertation:}

[2- leer]

[3] DEN ERLAUCHTEN, WOHLGEBORENEN, HOCHANGESEHENEN, HOCHERFAHRENEN, HOCHMÄCHTIGEN UND WOHLWEISEN HERREN

STÄTTMEISTERN, FÜNFERRÄTEN, UND RÄTEN

DER FREIEN STADT

DES HEILIGEN RÖMISCHEN REICHES, DIE SCHWÄBISCH HALL

IST, SOWIE DEN ÜBRIGEN HERRN, DENEN

DAS WOHL DER STADT ANVERTRAUT IST UND AM HERZEN LIEGT, [4] DEN HOCHHERRLICHEN UND HOCHANGESEHENEN MITGLIEDERN DER EINGESCHRIEBENEN VÄTER,

SEINEN STETS GNÄDIGEN UND MIT HÖCHSTER EHRERBIETUNG

UNTERTÄNIG ZU BEGLEITENDEN

\section{HERREN MÄZENATEN}

UND

\section{PATRONEN,}

11 Andreas Zieger: Das Matrikelbuch des Haller Gymnasium illustre, 1673-1811. Schwäbisch Hall 2000, S. 64

12 Gerd Wunder: Die Bürger von Hall. Sozialgeschichte einer Reichsstadt 1216-1802 (FWFr 16). Sigmaringen 1980, S. 132, 137 und 292. 


\section{ÜBERGIBT, WEIHT UND WIDMET \\ DIESES MEDIZINISCHE INAUGURALE PROBESTÜCK \\ MIT DER SCHULDIGEN HOCHACHTUNG \\ DER SO GROSSEN NAMEN \\ DER SEHR ERGEBENE \\ VERFASSER. ${ }^{13}$}

[5]

[Ein Stich im Format 10 x 4,5 cm zeigt die vieltürmige Stadt Halle an der Saale, auf einem Spruchband überschrieben mit HALLE]

\section{PHYSIKALISCH-MEDIZINISCHE INAUGURALDISSERTATION ÜBER \\ DAS FOSSILE SCHWÄBISCH HALLER ELFENBEIN VORWORT}

Daß Gott auch in unterirdischen Verstecken zu bewundern und zu verehren ist, zeigt die an Werken reiche Natur; und was wir in der Heiligen Schrift geoffenbart lesen, finden wir nicht selten durch die innersten Eingeweide der Erde bestätigt. Es führte zu weit, diese These mit ausführlichen Argumenten zu bestätigen, und es wird ausreichen, wenn wir nur mit wenigen Worten zeigen, daß nicht wenige Belege für eine allgemeine Sintflut im Schoß der Erde gefunden werden. Es wird niemandem verborgen sein, daß das ganze Weltall, das der höchste Baumeister der Dinge begründete, vornehmlich aus drei Schöpfungskategorien zusammengesetzt ist, [6] nämlich der mineralischen, der vegetabilen und der animalischen, und es wird niemandem entgehen, daß die Gottheit jeder Kategorie bzw. jedem Reich seine Grenzen so bestimmt festgesetzt hat, daß es in keiner Weise möglich ist, sie ohne eine Zerstörung des eigenen Lebens und der Natur insgesamt zu überschreiten. Metalle, und alles, was dem Mineralreich zugerechnet wird, erhielten im Innern der Erde einen so festen Sitz, daß sie hinausgetreten, wenn man so sagen darf, ihr Leben und ihre Stärke verlieren. Und die Grenzen, die dem animalischen Reich zugeteilt sind, werden nur von den Elementen Luft und Wasser eingeschlossen. Wenn sie verlassen werden, wird alles Animalische nur tot oder zumindest zum Tod bestimmt in den Wohnort der Mineralien, den der Abgrund der Erde gewährt, aufgenommen, wenn man von den Maulwürfen absieht und den Regenwürmern in der Erde und gewissen Insekten, die na-

13 Die Wörter auf S. [3]-[4] sind in Majuskeln und teilweise auch kursiv gesetzt. Soweit innerhalb des Textes ab S. [5] der Dissertation einzelne Wörter oder Sätze eine solche Groß- oder Kursivschreibung erhielten, wurde diese in der Übersetzung beibehalten. Das betrifft vor allem die Namen der von Beyschlag zitierten wissenschaftlichen Autoren, die Zitate aus ihnen und die nach der Dissertation gedruckte, von dem Vorsitzenden Friedrich Hoffmann an Beyschlag gerichtete Stellungnahme zu dessen Dissertation (S. [30]-[32]). Mit der unterschiedlichen Schriftgröße lehnen wir uns an das Original an. Die Literaturangaben, die auch bei Beyschlag kursiv gesetzt sind, wurden nicht übersetzt; siehe dazu die Anmerkungen. 
türlich die Oberfläche der Erde durchkriechen. Jedoch entgegen diesen Gesetzen der Natur sind die tiefsten Stellen der Erde voll von tierischen Knochen, und zwar nicht selten von solchen Tieren, die, als sie lebten, sich in weit entfernten Regionen ernährten und jetzt von ihrer animalischen Weichheit in eine steinerne und mineralische Natur übergegangen sind. Unser Deutschland ist kein Platz für Elefanten und Schalentiere des Meeres; und doch werden sehr viele von diesen Tieren, teils ganz, teils in Teilen aus der Tiefe unserer Orte ausgegraben. Das bezeugen die Museen mit Kuriositäten der Natur, die voll von solchen selteneren Gegenständen sind; und die naturkundlichen Schriftsteller zählen sie noch auf. Woher aber kamen so große fremdländische Tiere und so große, die beinahe Monster sind, aus dem fernen Indien und dem weit entfernten Meer in die untersten Abgründe unserer Erde, die den Menschen kaum zugänglich sind? Diese Tiere und besonders die Schalentiere des Meeres sind der klarste Beweis, daß die Länder einst einer großen [7] Überschwemmung ausgesetzt waren, und zwar keiner nur oberflächlichen; dagegen spricht die sehr tiefe Lage der ausgegrabenen Tiere; und auch keiner nur partiellen, denn dem steht ihre fast in allen Regionen begegnende Präsenz entgegen; sondern einer universellen, tiefen und staunenerregenden. Auf eine solche Überschwemmung weist die Erforschung der Natur, die jedoch nicht weiß, was für eine Sintflut das war. Die Heilige Schrift aber beschreibt dieselbe ausführlich. Und zur Gruppe dieser zu den Höhlen der Erde verdammten Tiere gehört auch das so erwähnte fossile Elfenbein und Einhorn, das wir mit dem Segen des höchsten Gottes jetzt zu behandeln uns entschlossen haben. Denn für unseren Vorsatz gab eine günstige Gelegenheit die hier in Halle reichere Menge dieses Fossils, als Schwäbisch Hall sie mit seinem Fossil bot, dessen Geschichte, soweit sie uns bekannt wurde, wir nun mit wenigen Worten vorausschicken werden.

\section{GESCHICHTE DES IN SCHWÄBISCH HALL GEFUNDENEN FOSSILEN ELFENBEINS}

Hall in Schwaben, eine in einem engen Tal, das sehr hohe Berge und dichte Wälder überall umgeben, gelegene Stadt, die auf einem von einer salzigen Flüssigkeit durchströmten Boden errichtet worden ist (weshalb eben dort auch Kochsalz gesotten wird, dessen Beschaffenheit Herr Licentiat HEFFELMEYERUS in einer Dissertation, die 1731 zu Altdorf bei Nürnberg verteidigt wurde, ${ }^{14}$ erläuterte), hat nicht selten eine Menge fossilen Einhorns dargeboten, das aus tiefen Höhlungen der Erde ausgegraben worden war. So erzählen die Annalen, im Jahr

14 Johann Michael Heffelmaier: Dissertatio inauguralis medica sistens historiam salis quod Halae Suivorum coquitur. Altdorf 1731, [D]. Zu Johann Michael Heffelmeyer (auch Heffelmaier; 16951742), Apotheker, später Arzt in Schwäbisch Hall, vgl. Armin Wankmüller: Die Geschichte der Apotheken und des Apothekerwesens in der Reichsstadt Schwäbisch Hall. In: Armin Wankmüller (Hrsg.): Beiträge zur württembergischen Apothekengeschichte, Bd. IX, 160 S., 1970-1972. Tübingen, [D], S. 1-20, hier: S. 13. 
1494 nach Christi Geburt, als die Erde zur Erbauung des Ratshauses tief aufgegraben wurde, sei ein solches sehr großes und langes Horn ohne Äste gefunden worden. Man findet es erwähnt bei JOANNES LAURENTIUS BAUSCH, $t r$. de unicornu fossili pag. $180^{15}$, \& CRUSIUS, annal. Suev. Lib. 9. pag. 3. c. $5^{16}$

[8] Sodann wurde im Jahr 1605 nicht weit von der Stadt in einem Tal [Bühlertal], das die beiden Neunbronn \& Hochstadt [Hohenstadt] genannten Weiler voneinander trennt, aus der Erde ein Horn von riesiger Größe ausgegraben. Es ist kaum glaublich, wenn ich sage, daß es mehr als fünf Zentner ${ }^{17}$ wiegt; aber gestützt auf öffentliche Zeugnisse kann ich dies versichern: denn heute noch ist es in Hall in der St. Michaelskirche für jedermanns Augen in eisernen Banden aufgehängt zu sehen und in einem Bild daneben ist es lebensgetreu gezeichnet. Diesem Bild des Horns ist folgende Inschrift beigegeben:

Tausend Sechshundert und Fünff Jahr,

Den Dreyzehenden Februarii ich gefunden war,

Bey Neunbronn an dem Hällischen Land,

Am Bühler Fluß zur lincken Hand,

Samt grossen Knochen und lang Gebein,

Sag Lieber, was Arth ich mag seyn.

Im übrigen ist es innen hohl, etwas gebogen, vorne spitz und von einer aschgrauen Farbe wie die Knochen, die überall aus den Feldern ausgegraben werden; darüber werden wir später an der betreffenden Stelle handeln.

Auch heutzutage mangelt es dem Haller Boden nicht an solchen Fossilien. Denn nahe der Stadt ist eine Lehmgrube, die den Ziegeleien den geeigneten weiß-rötlichen Lehm liefert. ${ }^{18}$ Die Grube ist mehr als sechs Mann tief. Vermischt mit diesem Lehm finden sich verschiedene weiße, aschgraue, in kleine Stücke gebrochene Knochen von großen Tieren, die in unseren Orten ungewöhnlich sind, und auch viel versteinertes Holz, ganze Bäume und sehr viele Fragmente eines

15 Johann Lorenz Bausch: De unicornu fossili ad normam \& formam academiae naturae-curiosorum schediasma. In: Johann Michael Fehr: Anchora sacra vel scorzonera, ad normam \& formam academiae naturae-curiosorum. Jena 1666, [D], S. 169-204, hier S. 190 (nicht wie oben angegeben S. 180). Zu Johann Lorenz Bausch (1605-1665), Stadtarzt in Schweinfurt und Mitbegründer der Leopoldina, siehe: Neue deutsche Biographie, Bd. 1. Berlin 1953, S. 673 f.

16 Martin Crusius: Annales Suevici, 3 Bde. Frankfurt am Main 1595/96, [D], hier: S. 498. Beyschlag übernimmt diesen Literaturhinweis wortwörtlich aus Bausch 1666 (wie Anm. 15), S. 190. Die Angabe ,pag. 3“ findet sich schon bei Bausch; richtig ist: pars 3 (Teil 3).

17 Bei Beyschlag lesen wir quinque centenarios (fünf Zentner), das wird ein Übertragungsfehler oder ein Missverständnis sein, denn dieses Gewicht (5 Hällische Zentner sind $255 \mathrm{~kg}$ ) ist viel zu hoch. Morhard (1554-1631) schreibt in seiner Haus-Chronik unter dem Datum 1605: [das Elefantenhorn] hat bey $125 \mathrm{lb}$ [Pfund] gewogen deß hällischen centners, das heißt wohl: 125 Pfund bezogen auf den Hällischen Zentner als Grundmaß; 125 Pfund sind 63,7 kg. Siehe Johann Morhards Haller Haus-Chronik. Hg. vom Historischen Verein für Württembergisch Franken. Schwäbisch Hall 1962, S. 59.

18 Es dürfte sich um die Lehmgrube bei der Langenfelder Ziegelhütte vor dem Langenfelder Tor gehandelt haben; eine zweite Ziegelhütte stand im Lindach. Vgl. Karl Hillenbrand, Ziegler in und um Schwäbisch Hall (Schriftenreihe des Vereins Alt Hall 1). Schwäbisch Hall 1974, S. 11, und Flurkarte 1: 2500 von 1827 Blatt NO 5845. 
fossilen Einhorns. Um diese herum ist Sand, [9] der sich sehr kalt anfühlt und ihnen fest anhängt. Unter der Erde sind sie sehr schwer, und auf einen Ofen gelegt werden sie leichter als Holz.

Dazu ergab sich unlängst eine neue, aber sehr traurige Gelegenheit, im Haller Boden Elfenbein zu finden. Nachdem nämlich im Jahr 1728 im Monat August diese Stadt in einem schweren Brand, der nach Menschen Gedenken, von kleineren Bränden abgesehen, seit Gründung der Stadt der dritte ist, zu mehr als einem Drittel abgebrannt war, und danach in ihr Bürger waren, um unter anderen Häuser für die Gemeinde zu errichten, die für öffentliche Zusammenkünfte bestimmt waren, da erschien zwischen den Ruinen in der Tiefe der Erde, die bis zu einer Tiefe von $16^{1 / 2} \mathrm{Fu}$ [4,65 m] aufgegraben worden war, ein rötlicher Lehm, der getrocknet und gebacken Natronsalz lieferte. Zusammen mit ihm ist eine ungeheure Menge des sogenannten fossilen Einhorns nicht nur in kleineren, sondern auch in größeren Stücken ausgegraben worden. Es war glatt, aschenweiß und zerbrechlich. Eines dieser Stücke war 71/2 Fuß [2,1 m] lang und 12 Finger [etwa $25 \mathrm{~cm}$ ] dick, und immer noch unvollständig. Das ist die Geschichte des fossilen Elfenbeins, das in Hall in Schwaben zu verschiedenen Zeiten gefunden wurde. Aber auch Hall in Sachsen [Halle an der Saale] ist nicht ohne die genannten Fossilien, von denen sehr viele, und zwar verschiedenartige Knochen, Hörner und Zähne von verschiedenen Tieren im Museum des erlauchten VORSITZENDEN [Friedrich Hoffmann] vorhanden sind. Sie wurden zum größten Teil in der Umgebung von Halle gefunden. Vor allem übertrifft unter diesen das Übrige an Größe ein Elefantenzahn, der das Gewicht von 12 Pfund [sächs. Apothekergewicht: 4,2 kg; Krämergewicht: 5,6 kg] hat und den wir mit den Worten des exzellentesten SCHULTZ, ${ }^{19}$ so wie sie sich in Commerc. Litter. Norimb. anno 1732. pag. $405^{20}$ finden, so beschreiben: Dieser Zahn, auffällig durch alle Zeichen seiner Authentizität, wurde in einem Steinbruch von Espersted $t^{21}$ ausgegraben. Das Dorf ist drei Stunden [10] von hier entfernt an der Straße, die nach Querfurt führt. Es ist Teil der Grafschaft Mansfeld. Es wird dort ein Kalkstein gebrochen, der einem grauen Marmor ähnelt und viel für die Böden von Häusern und für die Konstruktion von Treppen verwendet wird. In der Mitte eines solchen Steines, 20 Fuß [5,6 m] tief, wurde dieser Zahn gefunden. Aber er wurde leider von unvorsichtigen Handwerkern in drei Teile zerbrochen. Die nebeneinander gelegten Fragmente messen 2 Ellen [1,14 m]. Jedoch der vordere Teil,

19 Johann Heinrich Schulze (1687-1744), ab 1722 Professor an der Universität Halle an der Saale, gilt als Begründer der Geschichtsschreibung in der Medizin, siehe Heidrun Karin Mitzel-Kaoukhov: Die Briefe Johann Heinrich Schulzes (1687-1744) an Christoph Jacob Trew. Erlangen 2011, Univ. Diss., [D].

20 Im folgenden Abschnitt (auch bei Beyschlag kursiv) zitiert Beyschlag, nicht immer wörtlich, aus: Johann Heinrich Schulze: Ex Litteris D. D. JO. HENR. SCHULZII, Halae [Halle an der Saale] $\mathrm{a}$ [nte] d[iem] V. Octobris scriptis [am Tag vor dem 5. Oktober geschrieben]. In: Commercium litterarium ad rei medicae et scientiae naturalis incrementum. Nürnberg 1732, [D], S. 405 f.

21 Heute Ortsteil von Bad Frankenhausen (Kyffhäuserkreis). 
der in eine stumpfe Spitze ausläuft, konnte nicht erworben werden. Unterdessen besteht die Vermutung, daß dieser Teil mindestens noch eine Viertel-Elle [14 $\mathrm{cm}]$ gemessen hat. Das Elfenbein selbst ist durch die Länge der Zeit und die Kraft der kalkigen Wässer so weich und zerbrechlich geworden, daß es leicht zerkleinert werden kann. Deshalb ließ der illustre Herr [Besitzer] ihn mit herumgegebenen Ringen aus Messing befestigen, damit er mit geringerer Gefahr aufbewahrt und mit den Händen behandelt werden kann. Neben ihm sind andere Knochen gefunden worden, die jedoch durch die Länge der Zeit so brüchig geworden und von den Handwerkern so übel zugerichtet worden sind, daß man nicht mehr entscheiden kann, welcher Art sie waren. Ein besseres Schicksal hatte nur ein Zahn, den ich für einen Backenzahn halte, der genügend unversehrt beigebracht wurde und der mit den übrigen [Fundstücken] aufbewahrt wird. Diese Fossilien gaben uns die Anregung, sie, speziell das fossile Elfenbein, etwas ausführlicher abzuhandeln, was Gott aus der Höhe fördern möge.

\section{$\S \mathrm{I}$}

Es sind verschiedene Namen, durch die dieses Fossil bezeichnet wird, und von diesen besonders: fossiles Einhorn, fossiles Elfenbein und fossiles Spodium [Knochenasche]. Der Grund für das Attribut „fossil“ ist leicht offenbar, da man den Gegenstand nicht hat, wenn er nicht aus der Tiefe der Erde ausgegraben wird [lat.: effodiatur, Compositum von fodere, fodi, fossus, - ausgraben]. Und Elfenbein wird es genannt, weil es nach Farbe und Form entweder identisch mit echtem Elfenbein ist oder dem Elefantenzahn am nächsten kommt. Von nicht wenigen wird es nicht ohne Grund [11] eben für einen Elefantenzahn gehalten, der in der allgemeinen Sintflut der ganzen Welt von den strömenden Wassern in das verborgene Innere der Erde verbracht worden ist. Daher kommt auch der Name Spodium. Denn Spodium bezeichnet, auch wenn es allgemein eine Asche bedeutet, vor allem eine metallische; in einem spezielleren Gebrauch bezeichnet es das durch die Gewalt des Feuers kalzinierte [gebrannte] Elfenbein. Da aber das fossile Elfenbein meist kalziniert und brüchig gefunden wird, nennt man es Spodium.

\section{$\S$ II}

Diese zwei Namen scheinen deshalb ganz zur Natur der Sache zu passen. Doch der dritte, Einhorn, wird hinsichtlich seines Ursprungs den Geschichten und Erdichtungen des Volkes geschuldet. Man sagt nämlich, daß unser Fossil, wenn es einem Horn nahe kommt, nichts anderes ist als das Horn des Tieres, das man für das seltenste hält, das Einhorn, das entweder selbst ausstarb und in der Tiefe der Erde begraben wurde oder in der Sintflut hier und dort untertauchte. Wenn wir die tierkundigen Autoren um Rat fragen, finden wir vier Arten von auf der Erde lebenden Einhörnern. Die erste ist das Rhinoceros, das mit einem zurückgebogenen und gekrümmten Horn ausgestattet als Feind der Elefanten genannt wird. 
Vgl. PLINIUS, hist. anim. L. 8. c. $20 .{ }^{22}$ Die zweite sind indische Rinder, vgl. auch PLINIUS, cap. 2. Die dritte sind indische Esel, über die zu lesen sind: ARISTOTELES, hist. anim. Lib. 2. cap. $1{ }^{23}$ AELIANUS, de anim. Lib. 4, cap. $52,{ }^{24}$ und die vierte ist der Origes, eine Ziegenart, die BARTHOLINUS, $t r$. de unicornu p. $65^{25}$ erwähnt. Über ihre Existenz gibt es keinen Zweifel, wenn man den Autoren glauben kann, wobei es nicht nur Ohren-, sondern auch Augen-

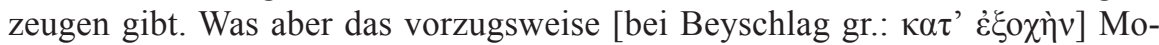
noceros bzw. Unicornu genannte Tier [12] betrifft, so erzählen die antiken Schriftsteller Unterschiedliches. Jeder von ihnen gibt eine besondere und sich von der des anderen unterscheidende Beschreibung, die fast nach einer erfundenen Geschichte riecht.

\section{$\S$ III}

Besonders lächerlich ist das, was die hebräischen Lehrer [im Talmud] über dieses Tier Monoceros [Einhorn] berichten. Rabbi Juda sagt in dem Buch Rabba, es sei so groß gewesen, daß Noah es nicht in seine Arche aufnehmen konnte. Rabbi Jacobi erzählt in seinem Kommentar zu $P s$ [alter] 22, v[ersus] $22,{ }^{26}$ daß David, als er damit beschäftigt war, seine Herde weiden zu lassen, zufällig auf ein schlafendes Einhorn gestiegen sei, da er es wegen der Größe von dessen Körper und Horn irrtümlich für einen Berg gehalten hatte. Als dann das Einhorn, aus seinem Schlaf erwacht, sich erhob, habe der erschreckte David, der auf dem Tier, das er für einen Berg gehalten hatte, saß, in an Gott gerichteten Gebeten ernstlich einen Tempel von einer Größe, die dem Horn des Einhorns gleichkam, nämlich von 100 Ellen, gelobt, wenn er ihn nur der gegenwärtigen Gefahr entrisse. Gott habe die Bitten erhört und, um das Einhorn zu erschrecken, einen Löwen ausgesandt, was die Furcht Davids so sehr vermehrt habe, daß er ausgerufen hätte: „Rette mich, Herr, aus dem Rachen des Löwen und entreiße mich den Hörnern der Einhörner!“”

\section{$\S \mathrm{IV}$}

Vielleicht boten diese und ähnliche Überlieferungen der Juden den Anlaß für die vielen Erfindungen von Einhorn-Sagen. Wenn wir aber die Autoren der Naturgeschichte aufschlagen, begegnen uns viele, die die Existenz der genannten Bestie verteidigen und ihre Gestalt beschreiben. Aus ihrer Zahl sind SOLINUS Poly-

22 Gaius Plinius Secundus: Historia Naturalis [nicht: animalium]; viele Drucke, z. B. Venedig 1469. Hier: Buch VIII, Kap. 29 [nicht 20].

23 Aristoteles: De historia animalium lib. 9. Venedig 1513, und später.

24 Claudius Aelianus: De natura animalium libri XVII. Lyon 1533, und später.

25 Thomas Bartholinus (1616-1680): De unicornu observationes novae. 2. Ausgabe Amsterdam 1645. 381 S., [D].

26 Psalm 22, Vers 22 nach der Übersetzung von Martin Luther, Wittenberg 1545: „Hilff mir aus dem Rachen des Lewen / Und errette mich von den Einhörnern“. 
hist. cap. 55, ${ }^{27}$ PLINIUS, nat. hist. Lib. 8. cap. $21,{ }^{28}$ AELIANUS, Lib. 16. de animal. cap. 20. und Lib. 17. cap. $44,{ }^{29}$ [13] ISIDORUS, Lib. 12. Etym. cap. $2,{ }^{30}$ GARCIAS AB HORTO Lib. 1. arom. hist. cap. $14^{31}$ und LUDOVICUS VARTOMANNUS Lib. 1. navigat. cap. 18, ${ }^{32}$ der versichert, selbst zwei Einhörner gesehen zu haben. Aber wegen der ganz verschiedenen Beschreibung dieses Tieres, die diese Autoren geben, ist es höchst wahrscheinlich, daß diese Autoren dieses Tier nie lebend oder tot gesehen haben. Deshalb zweifelten auch nicht wenige an seiner Existenz, unter denen es genügt, auf MERCURIALIS, ${ }^{33}$ tr. de febribus, Lib. 5., PARAEUS Lib. 20. cap. $39^{34}$ und SACHSIUS, in Monocerol. pag. 38, zu verweisen [im Original kursiv: allegasse sufficiat]. Wie dem auch immer sei, da von allen das Horn des Einhorn als schwarz beschrieben wird, das fossile Horn, das wir aus der Erde ausgegraben haben, aber weiß und aschefarben ist, folgt der sichere Schluß, daß es nicht von dem sogenannten Einhorn stammt.

\section{$\S \mathrm{V}$}

Nichtsdestoweniger würde ich aber nicht behaupten, der Name Monoceros sei leer und treffe auf keines der Tiere zu, sondern ich halte es mit den anderen Au-

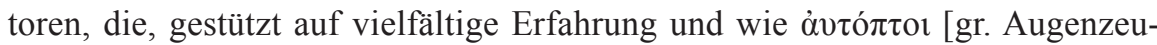
gen] bestätigen, das Monoceros, ein Tier und ein Meeresungeheuer von der Gattung der Wale, sei im Grönländischen Meer zu finden. Das bezeugen OLAUS MAGNUS Lib. 21. cap. 10, ${ }^{36}$ THOMAS BARTHOLINUS hist. anat. Lib. 4. cap. 4 und tr. de unicornu pag. 164, TULPIUS Lib. 4. obs. med. cap. $59^{37}$ und SACHSIUS Monocerol. pag. 26. seqq. [ff.], ${ }^{38}$ und sie liefern auch die Anatomie dieses Tieres. Diesem Tier schreiben sie ein sehr langes Horn zu, das mit einer Spitze versehen und gerade, hart, hohl und so weiß ist, daß es dem glänzendsten

27 Gaius Julius Solinus: Polyhistor, a Martino Anton. Delrio emendatus. Antwerpen 1572, Kap. 55, S. 222, [D]; andere Kapitelzählung in anderen Ausgaben.

28 Plinius (wie Anm. 22).

29 Aelianus (wie Anm. 24).

30 Isidorus: Etymologiae. Augsburg 1472 und später.

31 Garcias ab Horto (de Orta): Aromatum et simplicium aliquot medicamentorum apud Indos nascentium historia. Antwerpen 1574, [D].

32 Ludovicus Vartomannus (auch Ludovico de Varthema): Itinerario. Venedig 1535.

33 Vermutlich handelt es sich um Hieronymus Mercurial (auch Girolamo Mercuriale): Medici celeberrimri ac praestantissimi [...], medicina practica, libri V. Frankfurt am Main 1602, [D], hier: Buch 5, Kap. 1: De febribus in genere \& specie, S. $492 \mathrm{ff}$. .

34 Ambrosius Paraeus (auch Ambroise Paré): WundtArtzney/ oder Artzneyspiegell des Hocherfahrnen und Weitberühmbten Herrn Ambrosii Parei. Frankfurt am Main 1601, [D].

35 Paul Ludwig Sachse: Monocerologia, seu de genuinis unicornibus dissertatio. Ratzeburg 1676, [D].

36 Olaus Magnus: Historia de gentibus septentrionalibus. Rom 1555, hier: S. 739.

37 Thomas Bartholinus: Historiarunm anatomicarum. Centuria I-IV. Den Haag 1657, hier: S. 149 ff. und Bartholinus (wie Anm. 25); Nicolaus Tulpius: Observationes medicae. Editio nova. Amsterdam 1585, 382 S., hier: S. $372 \mathrm{ff}$.

38 Sachse (wie Anm. 35). 
Elfenbein sehr ähnlich zu sein scheint. Jetzt sind aber von den ausgegrabenen Einhörnern die, [14] die einst berühmter waren, wie das in St. Denis, in Venedig, ${ }^{39}$ in Antwerpen, der Schweiz, Utrecht u.s.w. dem genannten Horn des Walfisches in allem ähnlich, wie es SACHSE, dicto libro [a. a. O.] pag. 83 ausführlicher erläutert. Also ist es wahrscheinlich, daß die Fossilien, die überall im Schoß der Erde gefunden werden in der Form des beschriebenen Hornes, Zähne sind oder Hörner jenes Fisches, über den wir unten noch mehr sagen.

\section{$\S \mathrm{VI}$}

Wir kehren zu unserem Ausgangspunkt zurück und glauben, daran erinnern zu müssen, daß unter Elfenbein, Spodium und einem fossilen Einhorn jene härteren und aschenweißen Gegenstände verstanden werden, die den Knochen der Tiere völlig ähnlich aus tiefen Höhlen der Erde ausgegraben werden. Sie näher zu beschreiben, ist hier nicht der Ort. Sie finden sich zumeist in schlammiger und in sandiger Erde. Und was ihren natürlichen Ort anbelangt, so berichten die glaubwürdigen Autoren, da $ß$ er [der Ort] in vielen Reichen Europas gefunden wird. Besonders fossilträchtig aber ist jene Höhle im Harz nahe von Elbingerode, die man die Baumanns-Höhle ${ }^{40}$ nennt, wo sehr viele Fragmente fossilen Elfenbeins sowohl einst ausgegraben wurden als auch noch heute ausgegraben werden, von denen einige sehr kuriose im Museum des erlauchten Vorsitzenden betrachtet werden können. Vgl. auch GESNERUS, de lap figur. pag. 158, ${ }^{41}$ NEANDER, Physices Part. I. pag. $128,{ }^{42}$ FR. JOEL. Pract. T. V. Sect. 3. pag. $139{ }^{43} \mathrm{Da}$ unser Elfenbein auch in Thüringen ausgegraben wird, bezeugt LIBAVIUS part. 3 singular. L. 8. C. 17.44 In Meißen FABRICIUS annal. urb. Misnae L. $3,{ }^{45}$ in Hessen SCHROEDER pharm. med. cbym. l. 3 c. $4,{ }^{46} \mathrm{GESNER}, l[\mathrm{oco}]$ c [itato] pag. $254,{ }^{47}$ nicht weit von Speyer EISENMENGER, tr. de febrib. mal. pag. $36{ }^{48}$ weiter in Schwaben, der Schweiz, Böhmen, Mähren, Schlesien, Franken, Italien u.s.w.

39 Veneum im Druck der Dissertation ist ein Druckfehler für Venetum, das bei Sachse (wie Anm. 35), S. 83, steht.

40 Baumannshöhle: berühmte Tropfsteinhöhle in Kalksteinen des Devon bei Rübeland (Landkreis Harz) .

41 Konrad Gesner: De rerum fossilium, lapidum et gemmarum. Zürich 1565, [D], S. 158.

42 Michael Neander: Physice, sive potius syllogae physicae rerum eruditarum. Pars prima. Leipzig 1585, [D], S. 128.

43 Franziskus Joel (auch Franz Joël, 1508-1579): Operum Medicorum Joelis senioris Tomus quintus. Hrsg. von Franz Joel [1595-1631, der Enkel des oben genannten], Rostock 1629, [D], S. 139 .

44 Andreas Libavius: Singularium Pars Tertia. Frankfurt am Main 1601, [D].

45 Georg Fabricius: Rerum Misnicarum libri septem.; Annalium urbis Misnae lib. 3 [u. a.]. Leipzig 1569 (Leipzig 1598, Jena 1597), [D].

46 Johann Schroeder: Pharmacopoeia medico-chymica, sive thesaurus pharmacologicus. Ulm $1641 ;{ }^{2} 1644$ u. weitere; deutsch $1693,{ }^{2} 1709$ u. weitere, [D]; cbym. (statt chym.) ist ein Druckfehler. 47 Gesner (wie Anm. 41).

48 Samuel Eisenmenger (1534-1585), Mediziner und Theologe, war u. a. Leibarzt des Bischofs von Speyer; sein $\operatorname{tr}$ [actatus] de febrib[us] mal[is] konnte nicht ermittelt werden. 
[15] Vgl. BAUSCH, tr. de. Unicorn. pag. 189 seqq. [ff]. ${ }^{49}$ Auch in Halle in Sachsen fehlt in seinen Erdhöhlen dieser fossile Gegenstand nicht nach dem Zeugnis von Herrn D. LERCHE in seiner Dissertation über die Hallesche Ausgrabungsbeschreibung in diss. de Oryctographia Halensi, die er unter unserem erlauchten Vorsitzenden verteidigte; pag. $41 .^{50}$

\section{$\S$ VII}

An dieser Stelle ist eine besondere Bemerkung zu den Knochen nötig, die in Sibirien gefunden werden, über die uns die NOVELLAE PETROBURGENSES, besonders die ihnen hinzugefügten kritischen Besprechungen unterrichten. ${ }^{51}$ Auch in den ACTIS SUECIAE Tom. II. anno $1725^{52}$ wird dies nicht weniger ausführlich abgehandelt. Dort kommt aus dem Innern der Berge ein sehr ergiebiger Strom von Knochen in einer auffallenden Diversität. Es sind Schädeldecken, Wirbel, Rippen, Schienbeine, Armknochen und Zähne u.s.w., und zwar von einer solchen Größe, daß sie den Knochen von Elefanten sehr gleichkommen, ja sogar sie nicht selten übertreffen. Die meisten Knochen sind porös und leicht zerbrechlich, außer den Zähnen, die von so großer Härte gefunden werden, daß daraus Gefäße gemacht werden können, wie sie richtiges Elfenbein liefert. Die in den genannten NOVELLIS de anno 1730. pag. $323^{53}$ angeführte Größe eines Zahnes ist erstaunlich: er war schwerer als 280 Pfund und etwas gekrümmt, was auch bei den meisten anderen der Fall war.

\section{$\S$ VIII}

Es wird aber auch nicht unangebracht sein, aus denselben NOVELLAE hier die Meinung zu wiederholen, die die Einwohner Sibiriens über den Ursprung dieser Knochen befürworten. Sie glauben nämlich, daß es ein Tier gäbe von der Größe eines Elefanten, von schwarzer Farbe und mit zwei Hörnern ausgestattet, das, „Mammont“ genannt, nur in der Tiefe der Erde verweilen könne, da es der Luft [16] ausgesetzt sofort zugrunde gehe. Und sie glauben, daß von diesen Tieren die übergroßen Knochen stammen, besonders da sie durch ihr Alter verschieden und mehr oder weniger jung erscheinen. Wer sieht nicht, daß die Meinung irrig

49 Bausch (wie Anm. 15).

50 Johann Jakob Lerche: Dissertatio inauguralis physico-medica sistens oryctographiam Halensem sive fossilium et mineralium in agro Halensi descriptionem [...] April 1730. Halle a. d. Saale, $56 \mathrm{~S} .,[\mathrm{D}]$.

51 Es handelt sich um die deutschsprachige „St. Petersburgische Zeitung“ (St. Petersburgskie Vedomosti), die ab 1727 von der St. Petersburger Akademie der Wissenschaften zusammen mit einer Beilage (Petersburger Anmerkungen) herausgegeben wurde.

52 Acta Literaria Sueciae, Bd. II, 1725-1729. Uppsala und Stockholm, 614 S., hier: S. 36-43: Basilius Tatischow (auch Wassilij Nikitisch Tatistschew): Epistola (ad Benzelium) de Mamontowa Kost, id est de ossibus bestiae Russis Mamont dictae.

53 Von Wassilij Nikitisch Tatistschew (1686-1750) erschienen in den Beilagen zu den St. Petersburger Vedomosti (siehe Anm. 52) 1730 und 1732 einige Aufsätze zu den Mammutfunden in Sibirien und am Don. 
ist? Aber der hochgelehrte Kollege der Petersburger Gesellschaft, BAYERUS, ${ }^{54}$ zeigte hervorragend, daß der Ursprung dieser Geschichte ALCORANO TURCARUM [dem Koran der Türken] zugeschrieben werden muß, aus dessen 27. Surata [Sure] er eine seltsame Bemerkung von Mohammed über eine Bestie berichtet, die die Erde erforsche und am Ende der Welt aus der Erde herausgehen werde. ${ }^{55}$ Zugleich aber zeigt Bayer aus der Handschrift einer ruthenischen Chronik, daß die Tartaten [Tataren], die Mohammedaner waren, einst Sibirien bewohnt haben. Daher kam es zu dieser Überlieferung.

\section{$\S$ IX}

Nachdem wir dies in Parenthese hinzugefügt haben, kommen wir zu der schwierigen Frage, ob das Elfenbein zum animalischen oder zum mineralischen Reich gehört. Beide Auffassungen haben ihre Vertreter. Es gibt nämlich solche, die aufgrund der Form dieser Gegenstände, die die Knochen von Tieren exakt wiedergeben, sie dem animalischen Reich zuzählen wollten, aber da sie kaum begreifen konnten, wie die Tiere in eine solche Tiefe der Erde gelangten, wollten sie sie lieber dem Mineralreich zurechnen. Deshalb halten sie das fossile Elfenbein für eine Art Erde, die durch unterirdisches Wasser bzw. eine steinbildende Flüssigkeit aufgelöst worden und danach nach Verdunstung der feineren Materie in eine steinerne, zerbrechliche, verbrannten Knochen ähnliche Substanz zusammengewachsen ist. Sie vermuten aber, daß die so verschiedenen und so verwunderlichen Figuren einem wohltätigen Naturspiel zuzuschreiben sind; denn sowie jene Art von Erde noch in einem weicheren Zustand in diesen oder jenen Hohlraum in der Erde hineinfällt, vermehrt sie sich und wächst in jenem Hohlraum so lange, bis der Widerstand der sie umgebenden Erde viel stärker geworden ist, [17] und sie so den ursprünglichen Hohlraum ausfüllen und dessen Gestalt nachahmen. Diese Auffassung verteidigen BOETIUS A BOOT, ${ }^{56}$ OLAUS WORMIUS, ${ }^{57}$ BARTHOLINUS, ${ }^{58}$ BAUSCHIUS, ${ }^{59}$ und RHIEM, diss. de ebore fossil. Aldorff Noricorum anno 1682. habita $\$$. $16,{ }^{60}$ versucht, sie ausführlicher zu erklären. Als Beweis dienen ihnen die verschiedenen Figuren, die etwas Vegetabilem oder Animalischem gleichen und die in Steinen, von denen niemand behauptet, daß sie dem vegetabilen oder animalischen Reich entstammen, nicht

54 Gottlieb [Theophil] Siegfried Bayer (1694-1738), Historiker und Orientalist, Mitglied der St. Petersburger Akademie, veröffentlichte in der Zeitschrift der Petersburger Akademie „Commentarii Academiae scientiarum Imperialis Petropolitanae“, die ab 1728 in lateinischer Sprache erschien.

55 Koran, 27. Sure, Vers 81 nach Ibrahim Abou Nagie (Hg.): Die ungefähre Bedeutung des Al Qur‘ān Al Karīm. 5. Aufl., Köln 2012.

56 Anselmus de Boodt (auch Boëtius oder Boetius, 1550-1632): Gemmarum et lapidum historia. Hanau 1609 , [D].

57 Ole Worm (Olaus Wormius; 1588-1654): Historia rerum rarorum. Leiden 1655, [D].

58 Bartholinus (vgl. Anm. 25).

59 Bausch (vgl. Anm. 15).

60 Johannes Lucas Rhiem: Disputatio [nicht Diss.= Dissertatio] medica inauguralis de ebore fossili. Altdorf [nicht Aldorf] 1682, [D]. 


\section{De Fbore Foffilt Suevico Halenfi.}

ftentia, atque fic explere fuam matricem, ejusque figuram æmulari. Defendunt hanc opinionem BOETIVS A BOOT, OLAVS WORMIVS, BARTHOLINVS, BAVSCHIVS, eamque fufius explicare conatus RHIEM dilf. de ebore folfil. Aldorffi Noricorum anno 1682. babita S.16. Argumento illis funt variæ figuræ, vel vegetabile, vel animale aliquod æquantes, qux in lapidibus, quos nemo ex animali feu vegetabili regno generatos diceret, infigni arte inpreffie non raro deprehenduntur. Quidni igitur, putant, natura jocans lapides in figura cornuum ac dentium efformare poffet : eum illa, tefte AGRICOLA de nat. foffil. Li6.7. cap, 22, la-pidis arborum fimiles procrearet?

S. $X$.

WOLFFGANG WEDELIVS, Vir celebris, Progr. de ebore folfili, diftinctione quadam fibi confulturus, dividit unicornu fosfile in figuratum, \& formam dentis auc osfis cujusdam diftinctius exprimens; \& minus figuratum, aut irregulari forma præditum. Prius pro veriis animalium osfibus, longo tempore ad terram damnatis, onnino habet; alterum vero pro minerali marga, ae vera faxorum medulla, quæ mediae inter boIum \& cretam effet nature, venditat. Et deniqne BECHERVS, unicornu fosfile ex aqua fulphurea coagulata generari fcribit, Metallurg. part.t.pag.5.

6. XI.

Verum enim vero, juxta noftram fententiam, chymica horum fubjectorum analyfis, docere nos debet, cujusnam fint profapix, Prius vero, quam illam perquiramus; monere juvabit, figuras vegetabilium ac anima-

Abb. 3 Textprobe aus Beyschlags Druckschrift, S. 17; Druckexemplar des Stadtarchivs Schwäbisch Hall So 1726. 
selten mit besonderer Kunst eingedrückt angetroffen werden. Warum, so glauben sie, könnte die scherzende Natur nicht Steine in der Form von Hörnern und Zähnen ausformen, da sie nach dem Zeugnis von AGRICOLA de nat. fossil. Lib. 7. cap. $22,{ }^{61}$ aus Bäumen ähnliche Steine schuf.

\section{$\S \mathrm{X}$}

WOLFFGANG WEDELIUS, ein berühmter Mann, hat in seinem Progr. de ebore fossili ${ }^{62}$ sich mit einer gewissen Unterscheidung behelfend, das fossile Einhorn eingeteilt in das figürliche und die Form eines Zahnes oder Knochens deutlicher ausdrückende und das weniger figürliche oder mit einer irregulären Form versehene. Das erstere hält er durchaus für echte tierische Knochen, das andere aber empfiehlt er als eine mineralische Erdart und als das echte Innere von Felsen, das eine mittlere Natur zwischen einem Erdklumpen und einem Kalkstein habe. Und schließlich schreibt BECHERUS, Metallurg. part. I. pag. $5,{ }^{63}$ daß das fossile Einhorn aus schwefeligem Wasser zusammengewachsen entstehe.

\section{$\S \mathrm{XI}$}

Jedoch muß nach unserer Auffassung die chemische Analyse dieser Gegenstände uns lehren, zu welcher Sippschaft sie gehören. Bevor wir sie aber erforschen, wird es nützen, daran zu erinnern, daß diese Figuren von Vegetabilischem und Animalischem, [18] die Steinen eingeprägt sind, keineswegs ein Zeugnis für eine scherzende und spielende Natur bieten. Das ist so ferne, daß sie vielmehr bestätigen, daß echte Tiere und Pflanzen in die Tiefe der Erde hineingebracht wurden und sie die Urheber dieser Figuren sind. Wenn nämlich etwas Vegetabiles oder Animalisches, von Erde überschüttet, etwa in eine tuffsteinartige und noch weiche Materie hineinfällt und danach von einem steinbildenden Gas oder Wasser durchströmt wird, drückt es seine Spuren in seine Form, wird aber selbst durch die Länge der Zeit zersetzt. Diese Gegenstände nennt man überkrustet, und sie unterscheiden sich von den Versteinerungen, die Teile von Tieren oder Pflanzen sind, die wirklich in eine steinerne Substanz verwandelt worden sind. Jene werden in Tuffsteinen angetroffen, diese in Kalkgestein. Es ist jedoch nicht möglich, von diesen auf die Knochen zu schließen, die unter der Bezeichnung von fossilem Elfenbein zu finden sind. Daß sie ja keineswegs zum Mineralreich gehören, bestätigen ihre Ursprünge und andere Experimente.

\section{$\S$ XII}

Wenn wir nun aber also das fossile Elfenbein einer chemischen Analyse unterwerfen, wird es nicht anders als andere Knochen bei einer Destillation ein vergängliches trockenes und flüssiges Salz liefern und etwas von einem empyreu-

61 Georg Agricola: De natura fossilium libri X. Basel 1546.

62 Georg Wolfgang Wedel: Programma de unicornu et ebore fossili. Jena 1699, 16 S., [D].

63 Johann Joachim Becher: Metallurgia. Frankfurt am Main 1660. 
matischen [durch Verkohlung entstandenen] Öl. Wird es dann unter Einschluß verbrannt, so wird es schwarz werden. In offenem Feuer wird es sich in weiße, gläsern werdende Erde verwandeln, von der ein gewisses festes Salz herausgelöst werden kann, das mit Natron gemischt in einen alkalinischen Körper zurückgeführt wird; und wenn es [das Salz] mit einer geringen Menge von zerstoßenem Glas vereinigt wird, kann es in ein halbopakes milchiges Glas verwandelt werden, wie SAMUEL CARL in Lapide Lydia ${ }^{64}$ ausführlicher zeigte. Aber daß alle diese Phänomene nur zum Tierreich passen, ist das Ergebnis einer ständigen Beobachtung. Also wird niemand leugnen können, daß das fossile Elfenbein ein Abkömmling des Tierreichs ist. [19] Dabei ist es für uns nicht von Wichtigkeit, ob es figürlich oder weniger so ist. Dieses kann nämlich einst figürlich gewesen sein und ist vielleicht durch die Länge der Zeit oder die Nachlässigkeit der Ausgräber in mehrere Teile zerfallen. Indessen leugnen wir nicht, daß es figürliche Fossilien gibt, die gemeinhin als fossiles Elfenbein ausgegeben werden, aber in Wirklichkeit zur mineralischen Sippschaft gehören und den Namen einer Erdart oder des Innern von Felsen verdienen. Doch sie werden unpassenderweise als fossiles Elfenbein oder Einhorn bezeichnet.

\section{$\S \mathrm{XIII}$}

Da dies nun so ist, verstehe ich unter Elfenbein, Spodium oder fossilem Einhorn nichts anderes als einen knöchernen Teil irgendeines Tieres, der einst innerhalb der Erde verborgen wurde und in ihren tiefen Höhlen durch die beständige Ausdünstung der Wasser wissenschaftlich gesprochen kalziniert [in eine kalkähnliche Substanz umgewandelt] ist. Dieser Auffassung stimmt HOEHSTEDERUS, observ. medicin Dec. S. cas. 9 spodium ${ }^{65}$ zu, wenn er sagt: Ein Spodium ist nicht metallisch, sondern Teil irgendeines Knochens, der durch die Trockenheit der Erde ausgetrocknet ist, sei es, daß er ein Knochen oder Horn eines Elefanten oder eines anderen Tieres ist. Und wenn wir die Eigenschaften vergleichen, die zu diesem Gegenstand gehören, so werden wir finden, daß sie alle so gebildet sind, daß sie vor allem zu kalzinierten Knochen passen. Solche sind es 1. in Hinsicht auf die Konsistenz, daß der Gegenstand zerbrechlich ist, einem leichten Strich der Nägel nachgibt und mit Hilfe eines Mörsers leicht in Pulver zu versetzen ist, 2. in Hinsicht auf die Farbe, daß er weiß, aschig und mit schwarzen Punkten versehen ist; es ist bekannt, daß die Knochen der Lebewesen gänzlich weiß kalzinieren; 3. in Hinsicht auf den Geruch, nämlich daß er, in Kohlen geworfen, einen empyreumatischen tierischen Geruch abgibt, und Bausch beob-

64 Johann Samuel Carl: Lapis Lydius philosophico-pyrotechnicus: ad ossium fossilium docimasium analytice demonstrandam adhibitis et per multa experimenta chymico-physica in lucem publicam missus. Frankfurt am Main 1704. J. S. Carl (1677?-1757), in Öhringen geboren, hatte - wie später J. F. Beyschlag - bei Friedrich Hoffmann in Halle an der Saale Medizin studiert.

65 Johann Philipp Höchstetter: Rararum observationum medicinalium decades sex. Frankfurt am Main und Leipzig, 1674, [D]; hier: 5. Decade, Casus 9, S. 634. 
achtete in seinem Traktat über das fossile Einhorn ${ }^{66}$ daß er sehr angenehm sei, ähnlich dem, den Mandelmilch von sich gibt, 4. in Hinsicht auf den Geschmack, [20] daß er einen erdigen und kalkigen Geschmack bietet und an der Zunge kleben bleibt, und 5., daß er schließlich, in Wasser eingetaucht, zusammen mit ihm sprudelt. Alle diese Phänomene sind der Natur eines verbrannten oder kalzinierten Knochens keinesfalls fremd.

\section{$\S \mathrm{XIV}$}

Ich möchte jedoch nicht sagen, daß alle Knochen, die aus der Verborgenheit der Erde ausgegraben werden, immer kalziniert sind. Es gibt auch versteinerte. Aber ich stelle fest, daß diese nicht zur Klasse des fossilen Elfenbeins gehören. Außerdem gibt es im Grad der Kalzination große Unterschiede, denn einige sind so zerbrechlich, daß sie leicht in den Händen zerfallen können, andere aber werden härter und den rezenten fast ähnlich angetroffen, sodaß sie dem Ton [lat. limus] zugeordnet werden und aus ihnen Gefäße verschiedener Art gemacht werden können. Diese Differenz beruht auf einem dreifachen Grund: entweder hängt sie nämlich von der unterschiedlichen Natur des Geburtsortes oder der unterschiedlichen Konstitution des Knochens selbst ab oder schließlich von der Länge der Zeit, in der ein solcher Knochen in der Erde begraben war. So steht fest, daß sandige Orte für die Verkalkung von Knochen am geeignetsten sind und daß Zähne der Kalzination weniger ausgesetzt sind als andere Knochen.

\section{$\S \mathrm{XV}$}

Nachdem dies vorausgeschickt worden ist, folgt jetzt die entscheidende Frage, von welchem Tier die in der Erde zu findenden Knochen stammen. Nach meiner Meinung lassen sich die überall ausgegrabenen Knochen keineswegs auf ein bestimmtes Tier beschränken, sondern sie können von verschiedenen Tieren stammen. Es wurde oben gesagt, daß die, die sich in der Form eines länglichen Hornes zeigen, gemeinhin, aber fälschlich, dem Land-Einhorn zugeschrieben werden. Zumeist werden sie wegen ihrer Größe für Elefantenknochen gehalten. Warum? Weil das Volk nicht selten glaubt, die Skelette der Giganten oder Ena$\operatorname{ckim}^{67} \mathrm{zu}$ sehen. Aber Sicherheit wird in Fällen dieser Art [21] aus dem Vergleich der gefundenen Knochen mit der Beschreibung und den Skeletten anderer lebender Tiere gewonnen. Am häufigsten sind die Elefantenknochen, worin auch der Name des Elfenbeins seinen Ursprung hat. Sie erkennt man leicht, wenn sie mit der Anatomie des Elefanten verglichen werden, die A. MOULINUS in Dublin in Irland im Jahr 1681 gab und in englischer Sprache veröffentlichte. ${ }^{68}$ Nach

66 Bausch (wie Anm. 15).

67 Im Alten Testament (u. a. 4. Mose 13) genannte Riesen.

68 Allen Mullen (auch Moulin, Moulen): An anatomical account of the elephant accidentally burnt in Dublin, on Fryday, June 17, in the year 1681: Sent in a letter to Sir Will. Petty [...] with a relation of new anatomical observations in the eyes of animals [...]. London 1682. 
diesem Maßstab prüfte ERNESTUS TENTZELIUS in einer speziellen, an Antonio Magliabechi geschriebenen Episte ${ }^{69}$ die Figur eines ganzen, im Jahr 1696 im thüringischen Tonna ${ }^{70}$ ausgegrabenen Skelettes und begriff, daß dieses Skelett dem eines Elefanten vollkommen ähnlich war. Aber wer dürfte leugnen, daß außer Elefantenknochen auch die Knochen anderer Tiere zur Erde verdammt verborgen sind. Das Naturkundemuseum, das im Hallischen Waisenhaus eingerichtet ist, zeigt ein großes Horn, das einem polnischen Rind ähnlich ist. Es hing an einem vollständigen, weiß kalzinierten Skelett, das vor einigen Jahren im Sand bei Hornburg ${ }^{71}$ gefunden wurde. Nicht weit vom sächsischen Halle, im Dorf Vitzthal, wurde einmal das Horn eines Steinbocks ausgegraben, wie Herr D. LERCHE in allegata superius diss. pag. $41^{72}$ bezeugt. Über das Skelett eines Elchs, das in Masla gefunden wurde, hat HERMANN eine gelehrte Abhandlung verfaßt. ${ }^{73}$ Und was speziell diese überall gefundenen Hörner betrifft, so können sie, wenn sie mit der Beschreibung des Meeres-Einhorns übereinstimmen, die SACHSE in ejus Monocerologia ${ }^{74}$ gab und das er Narwhal nannte, durchaus für dessen Horn oder eher Zahn gehalten werden. Vgl. THOMAS BARTHOLINUS, tr. de Unicornu, pag. $112^{75}$ und OLAUS MAGNUS. ${ }^{76}$ So überliefert JOH. SEYFRID, medull. memorab. nat. fol. 593, ${ }^{77}$ daß im Jahr 1663 in Quedlinburg auf einem Feld ein vollständiges Skelett eines Einhorns mit erhobenem Kopf, [22] gefunden worden sei, an dessen Stirn ein mehr als vier Ellen [2,5 m] langes Horn gehängt habe.

\section{$\S \mathrm{XVI}$}

Schwieriger zu beantworten ist jedoch die Frage, wie die Knochen von für uns völlig fremdländischen Tieren in unser Land gekommen sind. HOECHSTEDERUS, ols. med. dec. V. cas. 9. pag. $651^{78}$ glaubt, daß dieses Fossil nicht durch ein Kunstwerk der Natur, sondern durch die Sorgfalt und Klugheit der Kaufleute ausgegraben werde. Und es ist auch nicht zu leugnen, daß es oft durch die Schlauheit von gewinnsüchtigen Fälschern geschieht, daß ein künstlich kalzi-

69 Wilhelm Ernst Tentzel: Epistola de sceleto elephantino Tonnae nuper effosso. Gotha 1696, [D]; ders.: Von dem zu Tonna ausgegrabenen Elephantencörper. Jena 1696, [D].

70 Gemeinde im thüringischen Landkreis Gotha.

71 Heute Ortsteil von Schladen-Werla, Landkreis Wolfenbüttel.

72 Übersetzt: In der oben angegebenen dissertatio S. 41 (siehe Anm. 50). Mit Vitzthal (so schon bei Lerche) ist Pfützthal gemeint, heute ein Ortsteil von Salzmünde (Saalekreis).

73 Leonhard David Hermann: Relatio historico-antiquaria de sceleto seu ossibus alcis Maslae detectis. Das ist: Historischer Bericht aus der Antiquitæt, von einem Elends-Thier-Cörper oder Knochen. Hirschberg 1729, [D].

74 Sachse (wie Anm. 35).

75 Bartholinus (wie Anm. 25).

76 Olaus Magnus (wie Anm. 36).

77 Johann Heinrich Seyfri[e]d: Medulla mirabilium naturae. Das ist Auserlesene/unter den Wundern der Natur/allerverwunderlichste Wunder/[...].Nürnberg 1679. Bei Beyschlag irrtümlicherweise memorab[ilium] statt mirabilium.

78 Siehe Höchstetter (wie Anm. 65); ols. (für obs.: observationum) ist ein Druckfehler. 
nierter Knochen eines Tieres tief in den Sand eingegraben und dann ausgegraben wird und als Kunstwerk der Natur und echtes fossiles Elfenbein teuer verkauft wird. Der hochexzellente Vorsitzende bezeugt selbst, daß auf diese Weise eben dies in jener unterirdischen Höhle, die man Baumannshöhle nennt, geschehen ist. Jedoch deshalb gleich die Existenz eines natürlich kalzinierten, fossilen Spodium gänzlich leugnen zu wollen, widerspricht der Erfahrung, da nicht wenige glaubwürdige Männer bezeugen, daß ein Spodium aus tiefen Verstecken der Erde ausgegraben wurde, die fast keinem Menschen zuvor zugänglich waren.

\section{$\S$ XVII}

Deshalb schien es mehreren der Gelehrten sehr wahrscheinlich, daß die genannten Knochen zur Zeit der universalen Sintflut unter der Erde verborgen wurden. Es lehrt ja die Heilige Schrift, daß zu dieser Zeit nicht nur der ganze Erdkreis überall von Wassern überflutet war, sondern auch, daß alle Landtiere, die Noah nicht in seine Arche eingeschlossen hatte, zugrunde gingen. Warum konnte also nicht geschehen, daß die Leichen der Elefanten und anderer Tiere aus Afrika und anderen entferntesten Gegenden der Erde [23] durch die reißenden Wasserströme nach Europa und in seine verschiedenen Regionen gebracht wurden? Diese Tiere, die schwerer als speziell die Erde waren, versenkten sich durch ihre riesige Größe, als die Wasser später abflossen, immer tiefer in sie. WEDELIUS Prog. de unicornu fossili pag. $15^{79}$ sagte in diesem Sinn: Denn nicht einmal die Erde konnte ihren Boden festhalten, ja sogar darüber hinaus erfuhr sie, zerstört, zerrissen und erschüttert, jene Beben, und die Worte reichen nicht aus, um dies zur Genüge auszudrücken, und durch jenen doppelten Druck des erzürnten Himmels und der sprudelnden, wildgewordenen Wasser erlitt sie die schrecklichsten inneren Bewegungen in ihren Eingeweiden und gewissermaßen in ihrem Zentrum; das Höchste war durch das Unterste, das Unterste durch das Höchste verwirrt und alles verstört und zerrissen. Und ich glaube, daß eben während dieser Sintflut es geschehen ist, daß die Monster des Meeres zu diesen oder jenen Orten gewälzt wurden und, als die Wasser allmählich austrockneten, dort verblieben.

\section{$\S$ XVIII}

Und es steht nichts dagegen, daß wir glauben, daß auch partikuläre Überschwemmungen der Erde in ältesten Zeiten hier und dort stattfanden und durch sie verschiedene Arten von Tieren, besonders einheimische, erstickt und in die Tiefe gestürzt wurden. Aber ich möchte auch nicht leugnen, daß sogar Menschen selbst absichtlich die Leichen von Tieren in verborgene Tiefen wälzten, die im Laufe der Zeit von Sand und Erde aufgefüllt wurden. Dazu kommt noch ein näherer Ursprung jenes Elfenbeins und fossilen Einhorns, das in Italien gefunden 
wird. Aus der römischen Geschichte ist ja bekannt, daß Hannibal einst sehr viele Elefanten nach Italien gebracht hat, die verstarben und schließlich der Erde anvertraut wurden. Vgl. CAESALPINUS, tr. de metallicis Lib. 2. cap. $40 .^{80}$ Was auch immer war, es dürften diese Fossilien in die Erde gekommen sein, woher sie immer [kommen] wollten: [24] genug für uns ist, daß sie grundsätzlich zum animalischen Reich gehören. Das bezeugt nicht nur ihre Form, sondern auch die chemische Analyse.

\section{$\S$ XIX}

Nach diesen Ausführungen sind spezieller ein paar Probleme zu betrachten, die in der Geschichte des bei Schwäbisch Hall ausgegrabenen fossilen Elfenbeins unsere Aufmerksamkeit verdienen. Und zwar stellt sich zuerst die Frage nach der Beschaffenheit jenes so genannten Hornes, das in der Kirche St. Michaelis aufgehängt ist. Daß es zur Sippschaft des Meereseinhorns gehört, widerlegt dessen Beschreibung von SACHSIUS, pag. $70,{ }^{81}$ jedenfalls, wenn jenes auf geradem Weg in einer Spitze endet. Unser Horn aber scheint, da es ein wenig gekrümmt ist, einen Bezug zu den Elefantenzähnen zu haben. Doch ich gestehe, daß uns von einer exakten Zuschreibung seine erstaunliche Größe abschrecken kann, die fünf Zentner gleichkommt. Indessen wollen wir probieren, ob wir aus den Zeugnissen der Autoren und der Analogie diese Größe irgendwie glaubwürdig machen können. Die ältesten Geschichtsdenkmäler über die großen Elefanten und ihre Zähne haben viele aufgezeichnet. ERASMUS FRANCISCI ${ }^{82}$ erinnert an einen Zahn dieser Art, der 150 Pfund [70,1 kg] wog, und VARTOMANNUS $^{83}$ sah in Sumatra zwei, die aufgehängt 336 Pfund [157 kg] wogen. Von nicht geringerem Gewicht müssen jene Zähne gewesen sein, die nach dem Zeugnis von PLINIUS ${ }^{84}$ und POLYBIUS ${ }^{85}$ an weit entfernten afrikanischen Küsten die Bewohner zu Pfosten und Pfählen benützten. Denn wenn auch Elefanten von der Größe, die die Zähne anzeigen, in unserer Zeit äußerst selten sind, ja sogar kaum vorkommen, so werden sie vielleicht doch in entfernteren Gegenden, die keiner der Unsrigen betrat, gefunden. [25] Im übrigen geben auch die Farbe und die zugespitzte und gekrümmte Form und die Hohlheit des Hällischen Horns an, daß man es mit Elefantenzähnen zu tun hat.

80 Andrea Caesalpino (auch Andreas Caesalpin): De metallicis libri tres. Rom 1696. Hannibal und die Elefanten werden in Kap. 48, nicht in Kap. 40 erwähnt.

81 Sachse (wie Anm. 35).

82 Erasmus Francisci: Neu-polirter Geschicht- Kunst- und Sitten-Spiegel ausländischer Völker [...]. Nürnberg 1670. Hier heißt es auf S. 1448: Man findet Zähne von ihnen [den Elefanten] / (aber keine Hörner / [...]) die zweyhundert Pfund / jedwedes Pfund zu zwölff Untzen unsers Gewigts gerechnet / wägen. Das entspricht etwa 150 württembergischen Pfund [70,1 kg].

83 Ludovico de Varthema: Itinerario de Ludovico de Varthema Bolonese nelle Egitto, [...].. Venedig ca. 1550, S. 67b. Dort lesen wir: CCC.\&. XXXV. lib., also 335 und nicht 336 Pfund ; das „," in „lib.“ wurde wohl als „eins“ angesehen.

84 Plinius (wie Anm. 22); hier: Buch VIII, Kap. 10.

85 Schon Plinius (wie Anm. 22) nimmt Bezug auf Polybios (um 200 v. Chr. bis um 120 v. Chr.). 


\section{$\S X X$}

Was die Fragmente des fossilen Spodium betrifft, die heute noch um Schwäbisch Hall herum ausgegraben werden, besonders die, die Lehmgruben liefern, so verdient vermerkt zu werden, daß alle sowohl die kalzinierten als auch die versteinerten Knochen, Hölzer ebenso wie Tiere meist angetroffen werden in kalkiger, lehmiger und sandiger Erde, da diese für jene Gegenstände am geeignetsten ist. Dagegen ruhen die verkrusteten Fragmente am ehesten in Tuffstein. Sodann ist erwähnenswert die Tiefe des Ortes, wo ausgegraben wird. In dieser Grube ist sie tiefer als die Länge von sechs Männern. Denn wir beobachten, daß dieser fossile Gegenstand meist sehr tief unter der Erde begraben ist. Es besitzt auch der erlauchte Vorsitzende einen gekrümmten Elefantenzahn, der in einem Esperstedt ${ }^{86}$ genannten Dorf nicht weit vom sächsischen Halle über einer Steinschicht 24 Ellen [15 m] tief in der Erde zusammen mit vielen anderen in Stücke geteilten Knochen gefunden wurde.

\section{$\S$ XXI}

[26] Dafür, daß die Fragmente von fossilem Elfenbein, die unter den Ruinen der durch den Brand zerstörten Häuser der Stadt gefunden wurden, ohne Zweifel solche waren, wird durch viele Anzeichen bezeugt. Erstens ist der rötliche Lehm, der Natronsalz lieferte, dessen Dunst manche steinbildend nennen, geeignet für die Begünstigung solcher Gegenstände. Die Zerbrechlichkeit und aschweiße Farbe bezeugen sodann ihre animalische Natur und daß einer [dieser Gegenstände], der von dem erlauchten Vorsitzenden in Kohlen geworfen wurde, einen tierischen Geruch von sich gab. Und es ist kein Zweifel, daß jenes große, siebeneinhalb Fuß [2,1 m] lange Stück, wenn es vollständig wäre, genauer gezeigt hätte, zu welcher Art Tier es gehört hat. Jedoch ist es eine berechtigte Vermutung, daß es ein Elefantenzahn ist, zumal da ein solcher noch kleinerer und jüngerer mit den übrigen Stücken an demselben Ort gefunden wurde. Im übrigen verdient noch Erwähnung, daß der Umstand, daß, wo unser Fossil gefunden wird, es nicht auf einen einzigen Ort beschränkt, sondern weithin verteilt ist, ein klarer Beleg dafür ist, daß an diesen Orten viele Tiere ihr Domizil hatten.

\section{$\S$ XXII}

Wir werden noch Weniges über den medizinischen Nutzen unseres Fossils hinzufügen und zuerst einiges über die Fähigkeiten des echten Einhorns aus den antiken Autoren exzerpieren. Denn sie priesen seine sehr große und beinahe magische Kraft gegen Gifte so sehr, daß es einst im Preis wie Gold geschätzt wurde. PHILOSTRATUS LEMNIUS, in vita Apollonii Thyanei L. 3. cap. $1^{87}$ \& AELI-

86 Siehe Anm. 21.

87 Flavius Philostratus: De vita Apollonii Tyanei libri octo. U. a. Venedig 1501-1504. Hier Buch 3, Kap. 2 (nicht Kap. 1). 
ANUS Lib. IV. de animal. cap. $52^{88}$ bezeugen, daß die Inder aus jenen Hörnern Pokale gemacht haben und berichten, daß die aus ihnen Trinkenden von keinen Krankheiten heimgesucht wurden und auch nicht vergiftet werden konnten. Weshalb ein Dichter sang: ${ }^{89}$

\section{Unoque [Beyschlag ed.: Unaque] in fronte superbi}

Cornu, quod Reges Indorum protinus aureis

Orbibus includunt, \& vina liquentia potant,

At tum nec morbos tuti [Beyschlag ed.: uti] sentire feruntur,

Nec quae inter mensas [Beyschlag ed.: menses] occculta hausere venena.

[und [die Einhörner sind] stolz mit einem einzigen Horn

auf der Stirn, das die Könige der Inder sofort in goldene Pokale

einschließen, und sie trinken [daraus] klare Weine,

und dann sollen sie sicher sein und keine Krankheiten spüren,

und auch nicht verborgene Gifte, die sie bei Tische zu sich nahmen.]

Aber wie die Existenz des Tieres selbst zweifelhaft ist, so sind auch die Kräfte dieses Hornes nicht vorhanden. Und wenn jenes Einhorn [27] auch wirklich existierte, könnten wir ihm keine andere Kraft zuschreiben als die, die das Horn eines Hirsches und rezentes Elfenbein besitzt.

\section{$\S$ XXIII}

Mit einer besseren Grundlage wird es deshalb erlaubt sein, die medizinische Wirkung des fossilen Einhorns zu erörtern. Einige Ärzte waren sehr damit beschäftigt, sie zu preisen. Sie erklären, daß es in seinen Wirkungen dem rezenten Horn des Einhorns ähnlich ist, besonders wenn es selbst noch rezent ist. Bemerkenswert ist die Geschichte, die PHILIPPUS HOECHSTETTERUS berichtet, der cum obs. med. decad. V. casu 9. p. $636^{90}$ schreibt: Wir gaben zwei Hunden von gleicher Statur eine Brechnuß in Milch für eine Drachme, einem von ihnen aber wenig später 12 Körner des Abriebs eines echten Einhorns in der gleichen Flüssigkeit. Beide erlitten verwunderliche Windungen und Zuckungen, von denen der eine, dem das Horn gegeben worden war, sich erbrach und leichtere Zuckungen verspürte, aber am dritten Tag lebendig und lebhaft entlassen wurde, der andere aber, der die Brechnuß eingenommen hatte, erlitt viele Zuckungen und starb innerhalb einer halben Stunde. Am folgenden Tag erfuhren wir die Kräfte desselben Horns an einem anderen Hund, der zwar viele Zuckungen

88 Aelianus (wie Anm. 24) berichtet im 4. Buch, Kapitel 52 von den wilden Eseln in Indien, die ein etwa 1 1 $1 \frac{2}{2}$ Ellen langes Horn auf der Stirn getragen haben sollen.

89 Es ist Petrus Angelius Bargaeus: Cynegetica. Item, carminum libri II, eclogae III. Lyon 1561, S. 41-42. Aus dessen Beschreibung des angeblichen indischen Einhorns in Buch 2 seiner hexametrischen Cynegetica werden hier mit drei oben verbesserten Druckfehlern die folgenden Verse zitiert. 90 Höchstetter (wie Anm. 65). 
hatte, aber keine tödlichen und der hier ohne Erbrechen wieder gesundete. Seit dieser Zeit habe ich zahllose Hörner, die als die eines echten Einhorns verkauft wurden, ausprobiert, aber sie blieben ohne jede Wirkung. usw. Meistens wird dieses Fossil als Heilmittel gegen Gifte, bösartige Fieber und die Pest sehr teuer verkauft, besonders vom Volk. Deshalb soll es für das Herz angenehm sein und dieses mit den übrigen erschlafften Eingeweiden stärken, von einem Gift kräftigen und diesem Widerstand leisten, die Pest vertreiben sowie Epilepsie, Ohnmacht und Herzrasen heilen.

\section{$\S \mathrm{XXIV}$}

Aber der zuvor gelobte HOECHSTETTERUS, loc. cit. [a. a. O.],[28] pag. 635, sagt bereits: Sie fabulieren über die riesige Wirkung dieses Spodium als ob es den Geist des Herzens reinige, stärke, bei Ohnmacht helfe, sich der Übelkeit widersetze und ganz die Kräfte hätte, die sie dem Horn eines Einhorns zuschreiben. Ich überlasse gerne diese Spodien, Knochen und Hörner den Reicheren und denen, die sich über Erfindungen freuen. Sie mögen ihnen vertrauen, so viel und so lange es ihnen beliebt. Mir gefällt mit Crato das Horn eines Hirschs. ${ }^{91}$ Und dieser Auffassung treten mehrere Ärzte bei. Sie ersetzen das fossile Elfenbein durch Hirschhorn oder durch den Knochen vom Herz des Hirsches ${ }^{92}$ oder durch rezentes Elfenbein, wie zu sehen ist bei FRANCISCUM de VERULAMIO in hist. vit: \& mort. pag. $191^{93}$ und JORDANUS de phaenom. pest. tr. 3. c. 9 . p. $594 .{ }^{94}$ Viele aber schreiben dem fossilen Elfenbein, besonders da es zerbrechlich ist, eine austrocknende und adstringierende Kraft zu, weshalb es nach dem Zeugnis von BOETIO â BOOT, tr. de lap. \& gemm. cap. $243^{95}$ bei Durchfall, Gonorrhö, weißem Ausfluß, zu großer Hämorrhagie [Blutung], Schlaffheit des Bauches und der Leber sowie zu tötenden Würmern gelobt wird; es soll äußerlich Geschwüre vernarben lassen, bei Verbrennungen und Wundsein nützen und, in feinstes Pulver zerrieben und mit Milch in die Augen geträufelt, Augentränen und Triefäugigkeit heilen.

\section{$\S \mathrm{XXV}$}

Wir stellen fest, daß nach unserer Meinung dieser Gegenstand nicht völlig ohne jeden medizinischen Nutzen ist. Aber mit HOFFMANN, in Clave ad Schroed. pag. $190,{ }^{96}$ ermahnen wir, daß wir ihn zuerst an Hunden erproben, da es leicht ist,

91 Beyschlag zitiert, mit geringfügigen Änderungen, Höchstetter (wie Anm. 65) S. 635 f. und 638.

Mit Crato ist Johann Crato von Krafftheim (1519-1585) gemeint.

92 Es handelt sich um die sogenannten Herzknochen des Hirsches, zwei kreuzförmige Knochen, die sich in der Scheidewand seiner Herzvorkammern bilden.

93 Franciscus Bacon de Verulamio (Francis Bacon): Historia vitae et mortis. Leiden 1636, [D].

94 Thomas Jordanus: Pestis phaenomena, seu de iis qui circa febrem pestilentem apparent exer-

citatio [...]. Frankfurt am Main 1576.

95 De Boodt (wie Anm. 56).

96 Friedrich Hoffmann: Clavis pharmaceutica Schroederiana. Halle an der Saale 1681. 
daß er von unterirdischen Ausdünstungen etwas Giftiges mit sich führt. Er hat am liebsten eine zartere erdige Substanz und ein Salzlager und paßt exakt mit Erdklumpen zusammen. Daher besitzt er eine leicht adstringierende und austrocknende Kraft, [29] und kann auch mit innerlich angewandten schweißtreibenden Pulvern und mit solchen, die allzu große Blutungen und Ausflüsse von Säften mäßigen, mit Erfolg vermischt werden. Was aber seine antiepileptische Wirkung betrifft, so haben wir da große Zweifel. Wenngleich nämlich Heilmittel, die aus dem Tierreich genommen wurden, eine spezifische Wirkung bei konvulsivischen und chronischen epileptischen Krankheiten haben, hat der erlauchte Vorsitzende jedoch durch häufige Erfahrung die Erkenntnis gewonnen, daß sie nur rezent dieses Lob verdienen und kein kalzinierter Knochen diese Wirkung hat. In der Tat, wenn es erlaubt ist, die Wahrheit zu sagen, können wir leicht ohne das fossile Elfenbein auskommen und können an seiner Stelle andere absorbierende, billigere und bereitliegendere Mittel, die zugleich eine schweißtreibende Kraft haben und die aus Tieren hergestellt und rezent gebrannt wurden, mit größerem Nutzen und geringerem Preis einsetzen.

[30]

\section{DEM EDLEN UND HERVORRAGEND GELEHRTEN HERRN KANDIDATEN DER VORSITZENDE.}

Daß eine genauere Kenntnis der natürlichen Dinge einen vernünftigen Arzt nicht nur schmückt, sondern vollendet, wird niemand in unserer Zeit leicht in Zweifel ziehen, wenn er diesem gar schönen Studium nicht völlig fremd ist. Es gibt viele, die die Natur nur durch Spekulationen und feinsinnige Ideen über die Anlage der Dinge kennen und schätzen. Andere aber bemühen sich, die Natur selbst zu beherrschen. Von dieser Art sind die, die aktiv werden und daran arbeiten, die Natur der körperlichen Dinge durch verschiedene Wirkungen, die chemische und mechanische Experimente zeigen, einzusehen und zu erklären, und sie strengen sich an, durch eine genaue Kenntnis der Phänomene, die mit den Gesetzen der Natur verbunden sind, die Werke Gottes zu imitieren. Und so müssen die sein, die in das innere Heiligtum der medizinischen Wissenschaft einzudringen begehren. Den antiken Ärzten fehlte die wirkliche physikalische und experimentelle Wissenschaft, und sie vernachlässigten auch die Wissenschaft des menschlichen Körpers und führten unter einem unglücklichen Stern anstelle von wahren Vorstellungen in die medizinische Lehre Termini ein, die nur die Ausgeburten der Ignoranz und Phantasie waren. Glücklicher ist darin aber unser Zeitalter, in dem durch Experimente und durch physikalische und anatomische Entdeckungen die geschicktesten Männer [31] die Heilkunst erstaunlich weit entwickelten und beleuchteten und unter Zurücklassung der Heimlichkeiten, die zur Ausübung der Heilkunst überhaupt nichts beitrugen, mit dem lobenswertesten Eifer daran arbeiteten, die Philosophie des menschlichen Körpers in die Form einer Wissenschaft zu bringen. Ich freue mich deshalb außerordentlich, wenn ich sehe, daß Rekruten in unserer Kunst in den 
Universitäten sich dem physikalischen und anatomischen Studium widmen. In diesem Kontext komme ich nicht umhin, von Herzen DIR, BERÜHMTESTER HERR KANDIDAT, zu gratulieren, da DU nicht nur heilsame Lehren, die dem praktischen Nutzen dienen, aus dem Mund der gelehrtesten Männer in unserer Universität durch ein unermüdliches Studium in dich eingesogen hast, sondern auch darüber hinaus mit nicht geringerem Fleiß eine Kenntnis der physikalischen und kuriosen Dinge DIR erworben hast, die besonders ich bezeugen kann. Denn wenn ich bis jetzt gewohnt bin, die mir im Alter gebliebenen Kräfte darauf zu verwenden, die jungen Studenten in diesem herrlichen Studium zu unterrichten, so hast DU an diesen Vorlesungen immer mit großem Lerneifer teilnehmen wollen. Ein Zeuge für eben diesen DEINEN Fleiß in den physikalischen Dingen stellt DEIN Inauguralstück dar, in dem DU das nicht weniger kuriose als nützliche Thema der unterirdischen Knochen zu behandeln DICH angestrengt hast, besonders des Elfenbeins bzw. des Elefantenzahns, der hier und da in Steinbrüchen ausgegraben wird, wie einer von riesiger und geradezu wunderbarer Größe in DEINEM Vaterland gefunden wurde. Es gibt immer noch mehrere, die diese Tierknochen als Reste der universalen Sintflut zu leugnen wagen, freilich ohne jede Grundlage. Wenn wir aber [32] die genaue Übereinstimmung ihrer Gestalt, Materie, Struktur und Form mit Tierteilen betrachten und darüber hinaus erwägen, daß sie in ein Feuer gegeben den Gestank eines flüchtigen öligen Salzes verbreiten und mit Hilfe eines großen Brennglases in eine völlig glasige Masse verschmelzen (und diese Phänomene sind nur bei Tierknochen anzutreffen, wie von mir in verschiedenen Experimenten beobachtet wurde, die ich mit einem Einhorn anstellte, das ich in meiner Sammlung seltener Gegenstände aufbewahre), dann werden wir von der Wahrheit der Sache und, daß es keinen Raum für einen Zweifel mehr gibt, mit hinreichender Klarheit überzeugt. Ich applaudiere deshalb dem von DIR erworbenen Verdienst, daß DU dieses ein wenig kuriosere und schwierigere Thema in dieser Dissertation zu bearbeiten unternommen hast. Ich habe nichts, was ich mehr wünsche, als daß vom glücklichsten Erfolg, wie DU ihn vier universitäre Jahre hindurch mit der Gnade und dem Segen Gottes in DEINEN Studien reichlichst erfahren hast, auch DEINE praktischen Versuche begleitet werden, so daß daraus auch die DEINEN und das dich nährende Vaterland und die armen Kranken die erwünschtesten Früchte erhalten können. 